\title{
Walks of molecular motors interacting with immobilized filaments
}

\author{
Theo M. Nieuwenhuizen ${ }^{a}$, Stefan Klumpp ${ }^{\mathrm{b}}$ and \\ Reinhard Lipowsky ${ }^{\mathrm{b}}$ \\ ${ }^{a}$ Instituut voor Theoretische Fysica, Universiteit van Amsterdam, \\ Valckenierstraat 65, 1018 XE Amsterdam, The Netherlands \\ ${ }^{\mathrm{b}}$ Max-Planck-Institut für Kolloid- und Grenzflächenforschung, \\ 14424 Potsdam-Golm, Germany
}

\begin{abstract}
Movements of molecular motors on cytoskeletal filaments are described by directed walks on a line. Detachment from this line is allowed to occur with a small probability. Motion in the surrounding fluid is described by symmetric random walks. Effects of detachment and reattachment are calculated by an analytical solution of the master equation. Results are obtained for the fraction of bound motors, their average velocity and displacement. Enclosing the system in a finite geometry (tube, slab) leads to an experimentally realizable problem, that is studied in a continuum description and also numerically in a lattice simulation.
\end{abstract}

Key words: molecular motors, random walks, lattice models, Brownian motion PACS: 87.16.Nn, 05.40.-a, 05.60.-k

\section{Introduction}

The interior of cells is both highly structured and dynamical. Active transport therefore plays a crucial role to target molecules to the various compartments of the cell as well as to maintain and reorganize the cell's structure. These tasks are accomplished by the cytoskeleton, a network of protein fibers, and the cytoskeletal motors, proteins which use the filaments of the cytoskeleton as highways or rails for directed transport.

Cytoskeletal motors are enzymes which catalyze the hydrolysis of adenosinetriphosphate (ATP) and, at the same time, use the free energy released from this reaction to perform mechanical work and convert it into directed movements. These motor molecules have been studied extensively during the last 
decade, both experimentally and theoretically. The main emphasis has been on the properties of single motor molecules, their motor mechanisms, and their directed walks along filaments $[1,2]$.

The nanometer size and piconewton forces of the molecular motors imply that the typical binding energies are of the order of the thermal energy $k_{\mathrm{B}} T$ and that the motor-filament binding can be overcome by thermal fluctuations. On large time and length scales $(\gg 1 \mathrm{~s}$ and $\gg 1 \mu \mathrm{m})$, motors perform peculiar random walks, where periods of directed active movements along filaments alternate with periods of non-directed Brownian motion in the surrounding fluid after unbinding from a filament $[3,4,5,6]$. (A related problem is the effective diffusion of an adsorbed particle along a surface via bulk excursions [7].) In order to study these random walks, we have recently introduced a class of lattice models, which are, on the one hand generic in the sense that they are independent of the specific motor mechanisms, but which we can, on the other hand, also apply to describe specific motor molecules by adapting the model parameters to the observed transport properties [4]. In addition, we can easily incorporate motor-motor interactions such as the mutual exclusion from binding sites of the filaments, which leads to 'traffic jam'-like density pattern and various kinds of phase transitions $[4,8,9]$

In the following, we will review our results for the motor's random walks obtained from these lattice models and present some new results from the corresponding continuum equations. The article is organized as follows: We introduce the lattice models in section 2. In section 3, we summarize our analytical results for the random walks on a lattice without confining walls. Compartments with confining walls which are experimentally accessible are studied in section 4, where we summarize our numerical results and present new analytical results from a continuum description of the random walks.

\section{Lattice models}

We have studied the random walks arising from many encounters with filaments by mapping them to random walks on a lattice $[4,5]$. A line of lattice sites represents a filament. Motors at these sites perform a biased random walk and move predominantly in one direction. Per unit time, they make a forward step, a backward step and no step with probability $1-\gamma-\delta / 2-\epsilon(d-1) / d$, $\delta / 2$, and $\gamma$, respectively, where $d=2,3$ is the spatial dimension. With a small probability $\epsilon / 2 d$, they move to each of the adjacent non-filament sites and thus unbind from the filament. At the non-filament sites the motors perform simple symmetric random walks and move to each neighbor site with probability $1 / 2 d$ ( $d$ denotes the spatial dimension) and rebind to the filament with probability $\pi_{\text {ad }}$ when they reach again a filament site. Confining walls are implemented as 
repulsive boundaries, at which all movements into the walls are rejected.

\section{The motors' random walks on a lattice}

Consider a discrete time random walk on a two dimensional square lattice with lattice sites labeled by integer coordinates $(n, m)$ with the above transition probabilities. The master equation for this dynamics reads $[5,6]$

$$
\begin{aligned}
P_{n, m}(t+1) & =\frac{1}{4} P_{n+1, m}+\frac{1}{4} P_{n-1, m}+\frac{1}{4} P_{n, m+1}+\frac{1}{4} P_{n, m-1} \quad(m \neq 0, \pm 1) \\
P_{n, 0}(t+1) & =\frac{1}{4} P_{n, 1}+\frac{1}{4} P_{n,-1}+\left(1-\gamma-\frac{\epsilon+\delta}{2}\right) P_{n-1,0}+\frac{\delta}{2} P_{n+1,0}+\gamma P_{n, 0} \\
P_{n, \pm 1}(t+1) & =\frac{1}{4} P_{n+1, \pm 1}+\frac{1}{4} P_{n-1, \pm 1}+\frac{1}{4} P_{n, \pm 2}+\frac{\epsilon}{4} P_{n, 0} .
\end{aligned}
$$

As initial condition we take an ensemble of particles at $n=m=0$. Note that, for simplicity, we have chosen the sticking probability $\pi_{\mathrm{ad}}=1$.

The Fourier-Laplace transforms of the probability distribution along the filament $P_{\mathrm{b}}(n, t) \equiv P_{n, 0}(t)$ and of the full distribution $P_{n, m}(t)$ are defined as

$$
P_{\mathrm{b}}(r, s) \equiv \sum_{t=0}^{\infty} \sum_{n=-\infty}^{\infty} \frac{e^{i r n} P_{n, 0}(t)}{(1+s)^{t+1}}, \quad P(q, r, s) \equiv \sum_{t=0}^{\infty} \sum_{m, n=-\infty}^{\infty} \frac{e^{i q m+i r n} P_{n, m}}{(1+s)^{t+1}}
$$

The master equations is reduced to an algebraic equation

$$
P(q, r, s)=\frac{1+\left[\gamma(1-\cos r)+\frac{1-\epsilon}{2}(\cos r-\cos q)+i v_{\mathrm{b}} \sin r\right] P_{\mathrm{b}}(r, s)}{s+1-\frac{1}{2} \cos q-\frac{1}{2} \cos r}
$$

where $v_{\mathrm{b}}=1-\gamma-\delta-\frac{1}{2} \epsilon$ is the average speed on the line. By integrating this result over $q$ we also obtain $P_{\mathrm{b}}(r, s)$ on the left hand side. It thus satisfies a linear equation, that is easily solved. We end up with the distribution

$$
P_{\mathrm{b}}(r, s)=\left[s+(1-\gamma)(1-\cos r)+\frac{1}{2} \epsilon\left(\cos r-e^{-\mu}\right)-i v_{\mathrm{b}} \sin r\right]^{-1}
$$

for the motors bound to the filament, where $\cosh \mu \equiv 2+2 s-\cos r$. The distribution for the unbound motors follows from Eq. (3). 


\subsection{Properties of the motors bound to the filament line}

Survival fraction. We extract the transport properties of the motor's random walk from (4). The value at $r=0$ gives the Laplace transform $N_{0}(s)$ of the probability $N_{0}(t) \equiv \sum_{n} P_{n, 0}(t)$ that the motor is bound to the filament:

$$
N_{0}(s)=\sum_{t=0}^{\infty} \frac{N_{0}(t)}{(1+s)^{t+1}}=[(1-\epsilon) s+\epsilon \sqrt{s(1+s)}]^{-1}
$$

For small times this implies $N_{0}(t) \approx 1-2 \epsilon \sqrt{t} / \sqrt{\pi}+\epsilon^{2} t$, which is a power series in $\epsilon \sqrt{t}$. For $t \ll 1 / \epsilon^{2}$, this is somewhat surprising: although the motors detach at times $\sim 1 / \epsilon$, the recurrent behavior of the random walk brings them mostly back to the filament, with half-integer powers in $t$ due to diffusion. For times $t \gg 1 / \epsilon^{2}$ we find $N_{0}(t) \approx\left[1-1 /\left(2 \epsilon^{2} t\right)\right] / \sqrt{\pi \epsilon^{2} t}$. The $t^{-1 / 2}$ decay expresses that finally all motors unbind, and agrees with scaling arguments $[3,4]$.

Average position and speed on the filament line. The expression (4) for $P_{\mathrm{b}}(r, s)$ contains much more information. At linear order in $r$ we may derive the average position of motor particles along the filament line, $N_{1}(t) \equiv \sum_{n} n P_{n, 0}(t)$. We obtain for short times $N_{1}(t)=v_{\mathrm{b}} t[1-8 \epsilon \sqrt{t} /(3 \sqrt{\pi})]$. The average position of the motors bound to the filament is given by $\bar{n}_{\mathrm{b}}(t) \equiv N_{1}(t) / N_{0}(t) \approx v_{\mathrm{b}} t(1-$ $2 \epsilon \sqrt{t} / 3 \sqrt{\pi})$ and their average speed is $\bar{v}_{\mathrm{b}} \equiv \mathrm{d} \bar{n}_{\mathrm{b}} / \mathrm{d} t \approx v_{\mathrm{b}}(1-\epsilon \sqrt{t} / \sqrt{\pi})$, where $v_{\mathrm{b}}$ is the average speed if the particles did not leave the line. For large times one gets $\bar{n}_{\mathrm{b}}(t) \approx v_{\mathrm{b}} \sqrt{\pi t}[1-2 /(\epsilon \sqrt{\pi t})] / \epsilon$ and $\bar{v}_{\mathrm{b}}(t) \approx v_{\mathrm{b}} \sqrt{\pi} / 2 \epsilon \sqrt{t}=(\pi / 2) N_{0}(t) v_{\mathrm{b}}$, confirming the scaling $\bar{v}_{\mathrm{b}}(t) \sim v_{\mathrm{b}} N_{0}(t)[3,4]$. The effective motor velocity is reduced by a factor $\sim N_{0}(t)$, i.e., by the probability that a motor is in the bound state. The relation $\bar{v}_{\mathrm{b}} \sim N_{0} v_{\mathrm{b}}$ also applies to a simple two-state random walk, where motion is directed in one of the states only. In contrast to the simple two-state random walk, however, the probability $N_{0}$ is time-dependent.

Dispersion and diffusion coefficient on the filament line. From the second moment $N_{2}(t)=\sum_{n} n^{2} P_{n, 0}(t)$ we may define the normalized second moment ${\overline{n^{2}}}_{\mathrm{b}} \equiv N_{2}(t) / N_{0}(t)$, the dispersion $\Delta n_{\mathrm{b}}^{2} \equiv{\overline{n^{2}}}_{\mathrm{b}}-\bar{n}_{\mathrm{b}}^{2}$ and the diffusion coefficient, $D_{\mathrm{b}}(t) \equiv(1 / 2) \mathrm{d} \Delta n_{\mathrm{b}}^{2} / \mathrm{d} t$. The results for the latter read at short $t$

$$
D_{\mathrm{b}}(t) \approx \frac{1}{2}(1-\gamma)+\frac{1}{6 \sqrt{\pi}} \frac{v_{\mathrm{b}}^{2}}{\epsilon^{2}} \epsilon^{3} t^{3 / 2}+\frac{2 \gamma-1}{4} \frac{\epsilon \sqrt{t}}{\sqrt{\pi}} .
$$

and for large $t$

$$
D_{\mathrm{b}}(t) \approx \frac{v_{\mathrm{b}}^{2}}{2 \epsilon^{2}}\left(4-\pi-\frac{\sqrt{\pi}}{\epsilon \sqrt{t}}\right)+\frac{(1-\gamma) \sqrt{\pi}}{4 \epsilon \sqrt{t}}+\frac{1}{4}
$$


The limiting value of the diffusion coefficient, $D_{\mathrm{b}}(\infty) \sim\left(v_{\mathrm{b}} / \epsilon\right)^{2}$ is large compared to the diffusion coefficient of the one-dimensional random walk along the filament, $D_{\mathrm{b}}(0)=(1-\gamma) / 2$. This broadening of the distribution occurs since the unbound motors lag behind the bound ones, which implies that also the rebinding motors lag behind those that have been bound for some time.

The density profile on the filament can be evaluated analytically for large $n$ and $t$,

$$
P_{n, 0}(t) \approx \frac{\epsilon n}{2 \sqrt{\pi v_{\mathrm{b}}}\left(v_{\mathrm{b}} t-n\right)^{3 / 2}} \exp \left(-\frac{\epsilon^{2} n^{2}}{4 v_{\mathrm{b}}\left(v_{\mathrm{b}} t-n\right)}\right)
$$

The exponential decay for $n \uparrow v_{\mathrm{b}} t$ expresses the large probability of unbinding for $t \gg 1 / \epsilon$. Comparison with the results of Monte Carlo simulations shows very good agreement for times larger than about 8000 time steps [6].

\subsection{Properties of the unbound motors}

Eventually every motor will unbind and diffuse in the surrounding fluid. We now discuss the effects of the filament on the behavior of the unbound motors.

Position and longitudinal diffusion. One finds for the average velocity of unbound motors at small times $\bar{v}_{\mathrm{ub}} \approx \frac{2}{3} v_{\mathrm{b}}\left(1-\frac{3}{8} \epsilon \sqrt{\pi t}\right)$ and at large times $\bar{v}_{\mathrm{ub}}(t) \approx v_{\mathrm{b}} /[\sqrt{\pi} \epsilon \sqrt{t}]$. Whereas each individual motor has zero average velocity in the fluid, the statistical velocity $\bar{v}_{\mathrm{ub}}$ is non-zero, since it is driven by unbinding from the cloud of motors moving on the filament. The longitudinal diffusion coefficient behaves at large times as $D_{\|}=(\pi-2) v_{\mathrm{b}}^{2} /\left[\pi \epsilon^{2}\right]+1 / 4$. The order of magnitude $D_{\|} \sim v_{\mathrm{b}}^{2} / \epsilon^{2}$ tells us that, like on the line, longitudinal diffusion is strongly enhanced by the unbinding from and binding back to the line. The perpendicular diffusion coefficient is modified much less, $D_{\perp}(t) \approx \epsilon \sqrt{t} /(2 \sqrt{\pi})$ at short times, while at large times $D_{\perp}(t) \approx 1 / 4-1 /(4 \epsilon \sqrt{\pi t})$. So the transverse diffusion starts out very small, and finally reaches its free space value.

The spatio-temporal density profile of the unbound motors can also be derived,

$$
P_{n, m}(t) \approx \frac{\epsilon\left(\epsilon n+2|m| v_{\mathrm{b}}\right)}{2 \sqrt{\pi v_{\mathrm{b}}}\left(v_{\mathrm{b}} t-n\right)^{3 / 2}} \exp \left(-\frac{\left(\epsilon n+2|m| v_{\mathrm{b}}\right)^{2}}{4 v_{\mathrm{b}}\left(v_{\mathrm{b}} t-n\right)}\right)
$$

The presence of multiple tracks on microtubules can be modeled by internal states, which keeps the problem solvable $[5,6]$. 
The same setup can be studied in $d=3[5,6]$. The Fourier-Laplace transformed density on the filament now reads

$$
P_{\mathrm{b}}(r, s)=\frac{3 I(r, s)}{\epsilon+\left[3(1-\epsilon) s+a\left(e^{-i r}-1\right)-b\left(e^{i r}-1\right)\right] I(r, s)} .
$$

with $a=\frac{1}{2}(\epsilon-3 \delta)$ and $b=\frac{1}{2}(6-6 \gamma-3 \delta-5 \epsilon)$ and involving the complete elliptic integral $I(r, s)=(1 / 2 \pi)^{2} \int_{0}^{2 \pi} \mathrm{d} q_{1} \mathrm{~d} q_{2}\left[3+3 s-\cos r-\cos q_{1}-\cos q_{2}\right]^{-1}$.

The main difference is now that the density of motors on the filament decays faster, as $\sim 1 / t$, because of a reduced return probability. Lack of space prevents us to discuss this any further, but we will present some results from continuum equations for the three-dimensional case below.

\section{Motor movements in confined geometries}

\subsection{Scaling arguments and numerical results}

We have also studied these random walks in compartments with simple geometries which are accessible to in vitro experiments [4]. In these geometries, a filament is immobilized to a surface, and the diffusion of unbound motors is restricted by confining walls. In the simplest case, the unbound motors can diffuse freely in the half space above the surface to which the filament is immobilized. By placing the filament in a quasi two-dimensional slab or in a rectangular or cylindrical tube, diffusion can be restricted along one or two dimensions perpendicular to the filament. We denote the linear extensions of the compartments by $L_{\perp}$. Scaling arguments and simulations show that, at large times with $t \gg t_{* *} \sim L_{\perp}^{2} / D_{\mathrm{ub}}$, when the motors experience the presence of the confining walls, these systems exhibit the same scaling behavior as systems with the same dimensionality, but without confining walls. For compartments with unconfined diffusion in $d_{\perp}$ dimensions perpendicular to the filament, the effective velocity is given by $v_{\mathrm{b}} N_{0}$ with $N_{0}(t) \sim t^{-d_{\perp} / 2}$.

\subsection{Continuum equations}

The continuum equations for the random walks of the motors can be solved in a similar way to the master equations of the lattice models. Since boundary conditions are easily implemented in the continuum model, we use them to derive analytical solutions for the half space, slab and tube geometry, see also

Ref. [10]. We denote the coordinate parallel to the filament by $x$ and the 
coordinates perpendicular to it by $\mathbf{y}=\left(y_{1}, y_{2}\right)$. The continuum equations are given by

$$
\begin{aligned}
\frac{\partial p}{\partial t} & =D_{\mathrm{ub}} \Delta p+\delta(\mathbf{y})\left[\tilde{\epsilon} P-\tilde{\pi}_{\mathrm{ad}} p_{0}\right] \\
\frac{\partial P}{\partial t} & =-v_{\mathrm{b}} \frac{\partial P}{\partial x}+D_{\mathrm{b}} \frac{\partial^{2} P}{\partial x^{2}}-\tilde{\epsilon} P+\tilde{\pi}_{\mathrm{ad}} p_{0},
\end{aligned}
$$

with the bound-state velocity and diffusion coefficient $v_{\mathrm{b}}$ and $D_{\mathrm{b}}$, the unbound diffusion coefficient $D_{\mathrm{ub}}, \tilde{\epsilon}=2 \epsilon / 3, \tilde{\pi}_{\mathrm{ad}}=2 \pi_{\mathrm{ad}} / 3$, and $p_{0}(x)=\ell^{2} p(x, \mathbf{y}=\mathbf{0})$.

Full three-dimensional space. Let us start with the full three-dimensional space. We use the Fourier-Laplace transformed form of Eqs. (11) and (12),

$$
\begin{aligned}
s p(r, \mathbf{q}, s) & =-D_{\mathrm{ub}}\left(r^{2}+q_{1}^{2}+q_{2}^{2}\right) p(r, \mathbf{q}, s)+\tilde{\epsilon} P(r, s)-\tilde{\pi}_{\mathrm{ad}} p_{0}(r, s) \\
s P(r, s) & =1+i v_{\mathrm{b}} r P(r, s)-D_{\mathrm{b}} r^{2} P(r, s)-\tilde{\epsilon} P(r, s)+\tilde{\pi}_{\mathrm{ad}} p_{0}(r, s),
\end{aligned}
$$

with the momentum $\mathbf{q}=\left(q_{1}, q_{2}\right)$ and with the same initial conditions as before. This leads to

$$
p_{0}(r, s)=\int_{-\infty}^{\infty} \frac{\mathrm{d} q_{1}}{2 \pi} \frac{\mathrm{d} q_{2}}{2 \pi} p(r, \mathbf{q}, s)=\frac{\tilde{\epsilon} P-\tilde{\pi}_{\mathrm{ad}} p_{0}}{2 \pi D_{\mathrm{ub}}} \int_{0}^{\infty} \mathrm{d} \tilde{q} \frac{\tilde{q}}{1+\tilde{q}^{2}} .
$$

To obtain a convergent integral, we introduce a cutoff $\tilde{q}_{c}$ as the upper integration limit. This cutoff corresponds to a certain range of attraction or thickness of the filament or a size of the motor and assures that the motor indeed returns to the filament. Defining

$$
I \equiv \int_{0}^{\tilde{q}_{c}} \mathrm{~d} \tilde{q} \frac{\tilde{q}}{1+\tilde{q}^{2}}=\frac{1}{2} \ln \left(1+\tilde{q}_{c}^{2}\right) \quad \text { with } \quad \tilde{q}_{c}=\sqrt{\frac{D_{\mathrm{ub}}}{s+D_{\mathrm{ub}} r^{2}}} q_{c}
$$

we obtain $p_{0}=\tilde{\epsilon} P /\left(2 \pi I^{-1}+\tilde{\pi}_{\mathrm{ad}}\right)$ and

$$
\begin{aligned}
P(r, s) & =\left(s-i v_{\mathrm{b}} r+D_{\mathrm{b}} r^{2}+\tilde{\epsilon}-\frac{\tilde{\epsilon} \tilde{\pi}_{\mathrm{ad}}}{2 \pi I^{-1}+\tilde{\pi}_{\mathrm{ad}}}\right)^{-1} \\
& \approx\left(s-i v_{\mathrm{b}} r+\frac{\tilde{\epsilon}}{\tilde{\pi}_{\mathrm{ad}}} \frac{4 \pi D_{\mathrm{ub}}}{\ln \left(D_{\mathrm{ub}} q_{c}^{2} s^{-1}\right)}\right)^{-1}
\end{aligned}
$$

where the second expression is valid for small $s$ and $r$. As before, the binding probability is obtained for $r=0$. For sufficiently small $s$, the cutoff term can be neglected, and inverting the Laplace transform leads to $N_{0}(t)=P(r=$ $0, t) \approx \tilde{\pi}_{\mathrm{ad}} /\left(4 \pi \tilde{\epsilon} D_{\mathrm{ub}} t\right)$ in agreement with the result from the lattice model. 
Half space. The case of the half space can be treated by the method of reflection and superposition, see e.g. Ref. [11]. The part of the three-dimensional solution for $y_{2}<0$ can be reflected at the surface, so that the superposition $p\left(y_{2}\right)+p\left(-y_{2}\right)$ is obtained, which fulfills the boundary condition $\partial p / \partial y_{2}=0$ at $y_{2}=0$. The density profile is symmetric under reflection at the plane $y_{2}=0$, so we obtain just twice the solution for the full three-dimensional space and thus, also the double for the velocity and the displacement.

Slab. Let us now consider a slab of height $2 L_{\perp}$, where the filament is located at $y_{1}=y_{2}=0$ in the middle of the slab. The case where the filament is immobilized at the lower surface of the slab follows then by reflection and superposition. The boundary conditions are $\partial p / \partial y_{2}=0$ at $y_{2}= \pm L_{\perp}$ or, equivalently, periodic boundary conditions at $y_{2}= \pm L_{\perp}$. We can therefore expand the unbound density into a Fourier series, $p\left(\ldots, y_{2}\right)=\sum_{j=-\infty}^{\infty} p\left(\ldots, \omega_{j}\right) e^{i \omega_{j} y_{2}} /\left(2 L_{\perp}\right)$ with $\omega_{j}=\frac{2 \pi j}{2 L_{\perp}}$. Here and in the following we use the symbol $\omega$ for discrete and $q$ for continuous momentum variables. For the other variables, we take the Fourier and Laplace transforms in the same way as above. Eq. (15) becomes now

$$
p_{0}(s, r)=\int_{-\infty}^{\infty} \frac{\mathrm{d} q_{1}}{2 \pi} \frac{1}{2 L_{\perp}} \sum_{j=-\infty}^{\infty} p\left(r, q_{1}, \omega_{j}, s\right) .
$$

We integrate out $q_{1}$ as before. In addition, we approximate the summation over $j$ by the term for $j=0$ and twice the integral

$$
S(r, s) \equiv \sum_{j=1}^{\infty} \frac{1}{\sqrt{s+D_{\mathrm{ub}} r^{2}+D_{\mathrm{ub}} \omega_{j}^{2}}} \simeq \frac{L_{\perp}}{\pi} \int_{\pi / L_{\perp}}^{\omega_{c}} \frac{\mathrm{d} \omega}{\sqrt{s+D_{\mathrm{ub}} r^{2}+D_{\mathrm{ub}} \omega^{2}}}
$$

where we introduced again a cutoff $\omega_{c}$. We obtain

$$
P(r, s) \approx\left[s-i v_{\mathrm{b}} r+\tilde{\epsilon}-\tilde{\epsilon}\left(1+\frac{4 L_{\perp} D_{\mathrm{ub}} / \tilde{\pi}_{\mathrm{ad}}}{\sqrt{\frac{D_{\mathrm{ub}}}{s+D_{\mathrm{ub}} r^{2}}}+\frac{2 L_{\perp}}{\pi} \sqrt{D_{\mathrm{ub}}} S(r, s)}\right)^{-1}\right]^{-1}
$$

for small $r$. For motors which have not yet reached the boundaries of the slab, i.e. for $s \gg D_{\mathrm{ub}}\left(\pi / L_{\perp}\right)^{2}$, we recover the result for the full three-dimensional space. For larger times or $s \ll D_{\mathrm{ub}}\left(\pi / L_{\perp}\right)^{2}$, the square root term is dominating in Eq. (20) and we obtain the two-dimensional result [10] with an effective reattachment rate $\tilde{\pi}_{\mathrm{ad}} /\left(2 L_{\perp}\right)$. In summary, at large times the probability to be bound to the filament and, thus, the effective velocity $v_{\mathrm{b}} N_{0}$ behave as

$$
N_{0}(t) \approx\left\{\begin{array}{cc}
\tilde{\pi}_{\mathrm{ad}} /\left(4 \pi \tilde{\epsilon} D_{\mathrm{ub}} t\right) & \text { for } t \ll t_{* *} \\
\tilde{\pi}_{\mathrm{ad}} /\left(4 L_{\perp} \tilde{\epsilon} \sqrt{\pi} \sqrt{D_{\mathrm{ub}} t}\right) & \text { for } t \gg t_{* *}
\end{array}\right.
$$


with the crossover time $t_{* *}=L_{\perp}^{2} /\left(\pi D_{\mathrm{ub}}\right)$.

Tube. Finally, a tube with quadratic cross-section can be studied in the same way. In this case we have two discrete momenta $\omega_{1, i}$ and $\omega_{2, j}$ corresponding to the transverse coordinates $y_{1}$ and $y_{2}$, and Eq. (15) is now replaced by

$$
p_{0}(s, r)=\frac{1}{\left(2 L_{\perp}\right)^{2}} \sum_{i, j=-\infty}^{\infty} p\left(r, \omega_{1, i}, \omega_{2, j}, s\right) \text {. }
$$

As in the case of the slab, we approximate the double sum by the term for $i=j=0$ plus an integral with a cutoff $\omega_{c}$,

$$
p_{0}(s, r)=\frac{\tilde{\epsilon} P-\tilde{\pi}_{\mathrm{ad}} p_{0}}{\left(2 L_{\perp}\right)^{2}}\left(\frac{1}{D_{\mathrm{ub}} r^{2}+s}+\frac{L_{\perp}^{2}}{D_{\mathrm{ub}} \pi^{2}} \int_{\pi / L_{\perp}}^{\omega_{c}} \frac{2 \pi \omega \mathrm{d} \omega}{D_{\mathrm{ub}}\left(r^{2}+\omega^{2}\right)+s}\right),
$$

which leads to

$$
P(r, s) \approx\left\{s-i v_{\mathrm{b}} r+\frac{4 L_{\perp}^{2} \tilde{\epsilon}}{\tilde{\pi}_{\mathrm{ad}}}\left(\frac{1}{s}+\frac{L_{\perp}^{2}}{\pi D_{\mathrm{ub}}} \ln \frac{1+D_{\mathrm{ub}} \omega_{c}^{2} / s}{1+D_{\mathrm{ub}} \pi^{2} / L_{\perp}^{2} s}\right)^{-1}\right\}^{-1}
$$

for small $s$ and $r$. For $s \gg D_{\mathrm{ub}}\left(\pi / L_{\perp}\right)^{2}$, i.e., for motors which have not yet reached the boundaries, this expression yields again the solution for the threedimensional case without confining walls, and for larger times, it leads to

$$
P(r, s) \approx\left[s\left(1+4 L_{\perp}^{2} \frac{\tilde{\epsilon}}{\tilde{\pi}_{\mathrm{ad}}}\right)-i v_{\mathrm{b}} r\right]^{-1}
$$

and, therefore, to a constant probability to be bound to the filament, $N_{0}(t) \approx$ $1 /\left(1+4 L_{\perp}^{2} \tilde{\epsilon} / \tilde{\pi}_{\mathrm{ad}}\right)$, and a constant effective velocity $v=v_{\mathrm{b}} /\left(1+4 L_{\perp}^{2} \tilde{\epsilon} / \tilde{\pi}_{\mathrm{ad}}\right)$. The crossover to the long-time regime is governed by the crossover time $t_{* *}=$ $v_{\mathrm{b}} / v \times \tilde{\pi}_{\mathrm{ad}} /\left(\tilde{\epsilon} 4 \pi D_{\mathrm{ub}}\right) \approx L_{\perp}^{2} /\left(\pi D_{\mathrm{ub}}\right)$ where the last expression holds for large $L_{\perp}$ and agrees with the crossover time obtained for the slab.

\section{Summary}

Molecular motors exhibit peculiar random walks which arise from the repeated binding to and unbinding from filaments. We have obtained analytical results for these random walks in systems without and with confining walls by solving the master equations of lattice models and the corresponding continuum equations. Such random walks are relevant both for intracellular phenomena such 
as, for example, the 'slow transport' in axons [12] and for artificial systems in nanotechnology in which molecular motors are used as transporters [13].

\section{References}

[1] J. Howard, Mechanics of Motor Proteins and the Cytoskeleton, Sinauer Associates, Sunderland (Mass.), 2001.

[2] M. Schliwa, G. Woehlke, Nature 422 (2003) 759-765.

[3] A. Ajdari, Europhys. Lett. 31 (1995) 69-74.

[4] R. Lipowsky, S. Klumpp, T. M. Nieuwenhuizen, Phys. Rev. Lett. 87 (2001) 108101.

[5] T. M. Nieuwenhuizen, S. Klumpp, R. Lipowsky, Europhys. Lett. 58 (2002) 468474 .

[6] T. M. Nieuwenhuizen, S. Klumpp, R. Lipowsky, Phys. Rev. E 69 (2004) 061911.

[7] O. V. Bychuk, B. O’Shaughnessy, Phys. Rev. Lett. 74 (1995) 1795-1798.

[8] S. Klumpp, R. Lipowsky, J. Stat. Phys. 113 (2003) 233-268.

[9] S. Klumpp, R. Lipowsky, Europhys. Lett. 66 (2004) 90-96.

[10] S. Klumpp, Ph.D. thesis, Universität Potsdam (2003).

[11] J. Crank, The Mathematics of Diffusion, 2nd Edition, Oxford University Press, Oxford, 1975.

[12] L. Wang, C.-L. Ho, D. Sun, R. K. H. Liem, A. Brown, Nature Cell Biol. 2 (2000) $137-141$.

[13] H. Hess, G. D. Bachand, V. Vogel, Chem. Eur. J. 10 (2004) 2110-2116. 